\title{
Efeito do tamanho de partícula do volumoso e da freqüência de alimentação sobre o consumo e a digestibilidade em ovinos ${ }^{1}$
}

\author{
Effect of the forage particle size and the number of feedings on intake and digestibility \\ in sheep
}

\author{
GOMES, Silas Prímola ${ }^{2 *}$; BORGES, Ana Luísa Costa Cruz²; BORGES, Iran²; \\ MACEDO JÚNIOR, Gilberto de Lima ${ }^{3}$; SILVA, André Guimarães Maciel ${ }^{4}$; PANCOTI, \\ Carlos Giovani ${ }^{2}$
}

\author{
${ }^{1}$ Parte da tese de doutorado do primeiro autor. \\ ${ }^{2}$ Universidade Federal de Minas Gerais, Escola de Medicina Veterinária, Departamento de Zootecnia. \\ Belo Horizonte, Minas Gerais, Brasil. \\ ${ }^{3}$ Universidade Federal de Uberlândia, Faculdade de Medicina Veterinária e Zootecnia, Uberlândia, Minas \\ Gerais, Brasil. \\ ${ }^{4}$ Universidade Federal do Pará, Faculdade de Medicina Veterinária, Castanhal, Pará, Brasil. \\ *Endereço para correspondência: silaspg@hotmail.com
}

\section{RESUMO}

Avaliou-se o efeito de quatro tamanhos de partícula do volumoso e de dois manejos alimentares sobre o consumo, a digestibilidade aparente e o comportamento ingestivo em ovinos. Utilizaram-se 24 carneiros com peso vivo médio de $37,0 \mathrm{~kg}$, alimentados com relação volumoso:concentrado (V:C) 25:75\%. Foi utilizado o feno de Tifton- 85 como volumoso e um concentrado comercial. Adotou-se esquema fatorial $2 \times 4$ em delineamento inteiramente ao acaso (dois manejos de alimentação e quatro tamanhos de partícula) o que totalizou 6 animais por tratamento. Os tratamentos não afetaram as variáveis referentes ao consumo e à digestibilidade aparente. $\mathrm{O}$ consumo médio de matéria seca foi de $97,43 \mathrm{~g} / \mathrm{dia} / \mathrm{UTM}$ e a digestibilidade aparente da matéria seca média observada foi de $66,12 \%$. O consumo de fibra em detergente neutro foi de 47,22g/dia/UTM. O menor tamanho de partícula (2mm) apresentou os menores tempos gastos (minutos/dia) com a ruminação $(214,58)$ e com a atividade mastigatória total $(360,41)$. Os animais gastaram $423,48 \mathrm{~min} / \mathrm{dia} / \mathrm{kg}$ do consumo de fibra em detergente neutro ruminando e $676,19 \mathrm{~min} / \mathrm{dia} / \mathrm{kg}$ do consumo de fibra em detergente neutro com atividade mastigatória total. Os tempos gastos com a ruminação e com a atividade mastigatória total em ovinos são diminuídos com a redução do tamanho de partícula do volumoso. Os tamanhos de partícula do volumoso e a frequência de fornecimento da dieta não afetam o consumo e a digestibilidade aparente dos nutrientes em ovinos.

Palavras-chave: atividade mastigatória, fibra em detergente neutro, fibra fisicamente efetiva, ruminação

\section{SUMMARY}

Intake, apparent digestibility and ingestive behavior were studied in sheep fed four particle size of forage in two daily frequencies. Twentyfour sheep with live weight of $37.0 \mathrm{~kg}$ were studied in a completely randomized design in factorial $2 \times 4$ scheme (two feeding frequency and 4 particle sizes) totaling six animals per treatment. Tifton- 85 hay and a commercial concentrate were used with a ratio of $25: 75 \%$, respectively. The responses of intake and apparent digestibility were not modified with the treatments. Dry matter intake (DMI) was $97.43 \mathrm{~g} / \mathrm{day} / \mathrm{kgW}^{0.75}$ and DMDA was $66.12 \%$. The intake of neutral detergent fiber was $47.2 \mathrm{~g} /$ day $/ \mathrm{kgW}^{0.75}$. Smaller particle size $(2 \mathrm{~mm})$ had lower times of rumination (214.58minutes/day) and chewing activity (360.41minutes/day). Animals spent 423.48 minutes $/$ day $/ \mathrm{kg}$ of intake of neutral detergent fiber in rumination and 676.19 minutes/day/ $\mathrm{kg}$ of intake of neutral detergent fiber 
in chewing activity. The times spent with rumination and the total chewing activity in sheep decreased as the particle size of the forage was reduced. The particle size of the forage and frequency of feeding did not affect the intake and apparent digestibility in sheep.

Keywords: chewing activity, neutral detergent fiber, physically effective fiber, rumination

\section{INTRODUÇÃO}

O consumo e os coeficientes de digestibilidade aparente dos nutrientes são influenciados por vários fatores, como o teor de nutrientes, pelos efeitos associativos entre os alimentos, pela relação Volumoso:Concentrado e pelo processamento dos alimentos (ALVES et al., 2003; FURUSHO-GARCIA et al., 2003; SILVA et al., 2005; ZANINE \& MACEDO JÚNIOR, 2006; MACEDO JÚNIOR et al., 2007; SILVA et al., 2007; ARRUDA et al., 2008; MACEDO JÚNIOR et al., 2009). A redução do tamanho de partícula do volumoso pode elevar a taxa de passagem do alimento no trato gastrintestinal e acarretar aumento do consumo de matéria seca (MS) e de nutrientes. Outra alternativa para aumentar a taxa de passagem e o consumo seria elevar a proporção de concentrado na dieta, por ser alimento mais digestível que o volumoso (VAN SOEST, 1994). Porém, o efeito da redução do tamanho de partícula do volumoso da dieta e do manejo nutricional sobre o consumo e os coeficientes de digestibilidade aparente dos nutrientes tem sido pouco estudado em ovinos.

De acordo com o NRC (2007) a moagem da forragem tem pouco impacto sobre o consumo e a digestibilidade do alimento, apesar de, alguns trabalhos terem demonstrado alterações sobre estes parâmetros.
A diminuição do tamanho de partícula através da moagem fina aumenta a superfície de exposição ao ataque microbiano, o que incrementa a taxa de digestão das células vegetais potencialmente digestíveis. No entanto, a diminuição do tamanho de partícula não necessariamente eleva a digestibilidade do alimento devido ao aumento no consumo e no fluxo de digesta pelo trato gastrintestinal.

O comportamento ingestivo afeta diretamente $o$ atendimento às exigências de fibra por influenciar a taxa de ingestão, a efetividade da mastigação e ruminação e, consequentemente, $\mathrm{o}$ rumem. O tempo de ruminação é consideravelmente influenciado pelas características físicas da dieta, como tamanho de partícula (MERTENS, 1997; FRANÇA et al., 2009), e pelo manejo nutricional adotado, como número de refeições (MICHALETDOREAU \& DOREAU, 2001; DOREAU, et al., 2003; DEVRIES et al., 2005). O aumento na oferta do alimento pode estimular à ingestão MS, o que influencia a taxa de passagem através do trato gastrintestinal $\mathrm{e}$, consequentemente, a digestibilidade dos nutrientes (DEVRIES \& VON KEYSERLINGK, 2005).

Assim, foram avaliados os efeitos do tamanho de partícula do volumoso e de dois manejos alimentares sobre $\mathrm{o}$ consumo, a digestibilidade aparente dos nutrientes e o comportamento ingestivo em ovinos.

\section{MATERIAL E MÉTODOS}

O ensaio de digestibilidade aparente e de comportamento ingestivo foi realizado no Laboratório de Metabolismo e Calorimetria Animal, localizado na Escola de Veterinária da UFMG em 
Belo Horizonte (MG), nos meses de agosto e setembro de 2005. As análises laboratoriais foram realizadas nas dependências do Laboratório de Nutrição do Departamento de Zootecnia da EV-UFMG.

Foram utilizados nesta avaliação 24 carneiros machos, com peso vivo médio de $37,0 \mathrm{~kg}$, castrados e alojados em gaiolas de metabolismo, providas de cocho, bebedouro e saleiro. Foram realizados dois períodos experimentais, cuja fase de adaptação teve duração de 21 dias e a fase de coleta de dados e de amostras duração seis dias. Os animais foram vermifugados no início das atividades experimentais e pesados no início e no final de cada período. As temperaturas máximas e mínimas, durante $\mathrm{o}$ ensaio foram em média, respectivamente, $31^{\circ} \mathrm{C}$ e $21^{\circ} \mathrm{C}$ e os valores máximos e mínimos de umidade relativa do ar foram, respectivamente, $90,0 \%$ e $28,0 \%$, em média.

As dietas foram balanceadas de acordo com o NRC (1985) para ganho de peso com uma relação $\mathrm{V}$ :C $25: 75 \%$ na MS, e foram deixadas sobras de $5 \%$. Foi utilizado o feno de Tifton-85 (Cynodon spp.) como volumoso e concentrado comercial de manutenção constituído de milho moído (17,2\%), farelo de glúten de milho $(8,3 \%)$, gérmen de milho gordo $(15,0 \%)$, casca de soja $(24,0 \%)$, resíduo de feijão $(18,0 \%)$, farelo de babaçu $(12,0 \%)$, óleo vegetal $(1,0 \%)$, melaço líquido $(1,0 \%)$, cloreto de sódio $(0,6 \%)$, calcário calcítico $(2,8 \%)$ e premix mineral vitamínico $(0,1 \%)$, com o volumoso fornecido separadamente do concentrado. A composição bromatológica do volumoso, do concentrado e da dieta experimental encontra-se na Tabela 1 .

Tabela 1. Composição bromatológica do feno (FENO), do concentrado (CONC) e da dieta utilizada

\begin{tabular}{lrcc}
\hline Item & Feno & Concentrado & Dieta $^{2}$ \\
\hline Matéria seca (\%) & 89,45 & 87,91 & 88,30 \\
Matéria orgânica $^{1}$ & 94,11 & 92,18 & 92,66 \\
Proteína bruta $^{1}$ & 4,89 & 19,38 & 15,75 \\
Extrato etéreo $^{1}$ & 1,52 & 1,04 & 1,16 \\
Cinzas $^{1}$ & 5,09 & 9,13 & 8,12 \\
Carboidratos não fibrosos $^{1}$ & 10,44 & 32,21 & 26,77 \\
Fibra em detergente neutro $^{1}$ & 77,25 & 39,56 & 48,98 \\
Fibra em detergente acído $^{1}$ & 36,50 & 21,50 & 25,25 \\
Lignina $^{1}$ & 2,25 & 4,12 & 3,65 \\
\hline${ }^{1}{ }^{1}$ da ${ }^{2}{ }^{2}$ Dieta & & &
\end{tabular}

Foram adotados dois manejos alimentares denominados F2 e F4, respectivamente: duas refeições diárias às $7 \mathrm{hs}$ e $19 \mathrm{hs}$ ou quatro refeições diárias às $7 \mathrm{hs} ; 11 \mathrm{hs} ; 15 \mathrm{hs}$ e $19 \mathrm{hs}$. O feno foi submetido à desintegração com triturador comercial, dotado de peneiras com abertura de malhas de quatro tamanhos: $\mathrm{T} 1-2 \mathrm{~mm}, \mathrm{~T} 2-5 \mathrm{~mm}, \mathrm{~T} 3-$ $10 \mathrm{~mm}$ e T4 $-25 \mathrm{~mm}$; que constituíam respectivamente, os tamanhos de partícula denominados de TP2, TP5, TP10 e TP25. Aproximadamente 500g do material obtido em cada peneira foram colocados no separador de partículas modelo Penn State contendo 
Rev. Bras. Saúde Prod. Anim., Salvador, v.13, n.1, p.137-149 jan/mar, 2012 http://www.rbspa.ufba.br ISSN 15199940

duas peneiras (porosidades de 19 e $8 \mathrm{~mm}$ ) e uma bandeja de fundo, e avaliados de acordo com Lammers et al. (1996). O perfil de distribuição (\%) da fibra em detergente neutro (FDN) das partículas do feno e os diâmetros geométricos médios dos diferentes tratamentos são apresentados na Tabela 2. $\mathrm{O}$ fator de efetividade física (fef) dos diferentes tamanhos de partícula foi determinado como a fração da MS retida acima da peneira com porosidade de $8 \mathrm{~mm}$, e a fibra em detergente neutro fisicamente efetiva (FDNfe) obtida pela multiplicação do fef pelo teor da FDN da amostra de feno. $O$ diâmetro geométrico médio (Dgm) e o desviopadrão geométrico médio (Sgm) foram calculados conforme o ASAE (2001), citado por Kononoff \& Heinrichs (2003).

Tabela 2. Perfil de distribuição das partículas (\%) na matéria seca (MS) e na fibra em detergente neutro (FDN) em função dos tratamentos

\begin{tabular}{lrcrr}
\hline \multirow{2}{*}{ Tamanho de partícula } & \multicolumn{4}{c}{ Tratamento } \\
\cline { 2 - 5 } & TP2 & TP5 & TP10 & TP25 \\
\cline { 2 - 5 }$>19 \mathrm{~mm}$ & 0,00 & 0,00 & 17,81 & 40,18 \\
$8-19 \mathrm{~mm}$ & 0,00 & 35,35 & 14,42 & 14,88 \\
$<8 \mathrm{~mm}$ & 100,00 & 64,65 & 67,77 & 44,94 \\
Dgm $(\mathrm{mm})$ & 0,83 & 2,14 & 2,34 & 5,26 \\
Sgm $(\mathrm{mm})$ & 1,00 & 4,94 & 6,28 & 12,22 \\
\hline \multicolumn{5}{c}{ \% FDN retida } \\
\hline$>19 \mathrm{~mm}$ & 0,00 & 0,00 & 18,15 & 40,19 \\
$8-19 \mathrm{~mm}$ & 0,00 & 36,81 & 14,89 & 14,71 \\
$<8 \mathrm{~mm}$ & 100,00 & 63,19 & 66,96 & 45,10 \\
Fef & 0,00 & 0,37 & 0,33 & 0,55 \\
FDNfe $(\% \mathrm{MS})$ & 0,00 & 28,58 & 25,49 & 42,49 \\
\hline
\end{tabular}

TP2 = tamanho de partícula de $2 \mathrm{~mm}$; TP5 = tamanho de partícula de $5 \mathrm{~mm}$; TP10 = tamanho de partícula de $10 \mathrm{~mm}$; TP25 = tamanho de partícula de $25 \mathrm{~mm}$.

Dgm = diâmetro geométrico médio calculado conforme ASAE (2001); Sgm = desvio padrão geométrico médio calculado conforme ASAE (2001). Fef $=$ fator de efetividade física $[(100-\%$ de $\mathrm{MS}<8 \mathrm{~mm}) / 100]$; FDNfe $(\% \mathrm{MS})=$ fibra em detergente neutro fisicamente efetiva (fef $* \%$ FDN do feno $)$ expressa como porcentagem da matéria seca.

Durante a coleta foram pesados e amostrados diariamente o alimento oferecido, as sobras e as fezes e foi feito um pool das amostras para cada animal dos cinco dias coletados. As fezes foram recolhidas em bandejas plásticas e a urina acondicionada em baldes adaptados com tela separadora que continham $100 \mathrm{~mL}$ de ácido sulfúrico 2 N. As amostras de alimentos, sobras e fezes foram pré-secas em estufa ventilada a $55^{\circ} \mathrm{C}$ e moídas a $1 \mathrm{~mm}$, e a urina mantida congelada. Para as amostras de alimento oferecido, sobras e fezes foram realizadas as análises de MS a $105^{\circ} \mathrm{C}$, proteína bruta (PB), extrato etéreo (EE) e cinzas, conforme técnicas descritas por Silva \& Queiroz (2002). As análises de FDN e fibra em detergente ácido (FDA) foram realizadas 
de acordo com a metodologia de Van Soest et al. (1991). Nos alimentos oferecidos também foi determinado o teor de lignina, conforme técnica descrita por Silva \& Queiroz (2002). Os coeficientes de digestibilidade aparente foram calculados a partir da relação entre o consumo e a excreção fecal dos nutrientes. $\mathrm{O}$ cálculo do teor de matéria orgânica (MO) foi feito pela diferença entre o teor de MS e de cinzas.

A concentração de carboidratos não fibrosos (CNF) dos alimentos foi obtida pela equação: $100-(\% \mathrm{FDN}+\% \mathrm{~PB}+$ $\%$ EE + \%cinzas), de acordo com Weiss (1999). Os teores de nutrientes digestíveis totais (NDT) foram calculados por meio da equação de Weiss (1999): NDT $=$ PBD $+(2,25 \mathrm{x}$ EED) + FDND + CNFD, em que $\mathrm{PBD}=$ proteína bruta digestível; $\mathrm{EED}=$ extrato etéreo digestível; $\mathrm{FDND}=$ fibra em detergente neutro digestível; $\mathrm{CNFD}=$ carboidratos não fibrosos digestíveis.

Para o estudo de comportamento ingestivo, os animais foram observados durante 24 horas ininterruptas, no primeiro dia do período de coletas, e a cada cinco minutos eram feitas anotações sobre a atividade dos animais. As variáveis analisadas foram: tempos de ingestão de água e de sal, em ócio, ruminação, ingestão de ração e atividade mastigatória total (tempo de ruminação + tempo de ingestão de ração). Para quantificar o tempo de cada variável em minutos multiplicou-se $\mathrm{o}$ número de observações por cinco.

Os dados foram avaliados por meio de análises de variância com a utilização do SAEG 8.0. Adotou-se esquema fatorial $2 \times 4$, duas frequências de alimentação e quatro tamanhos de partícula do feno, em delineamento inteiramente ao acaso, com seis repetições (animais), o que totalizou 24 animais. Para comparação das médias das variáveis foi utilizado o teste SNK, em nível de $5 \%$.

\section{RESULTADOS E DISCUSSÃO}

Não houve interação entre os diferentes tamanhos de partícula e a frequência de alimentação, que não afetaram as variáveis referentes ao consumo de nutrientes $(\mathrm{P}>0,05)$.

Os consumos médios de matéria seca (CMS) em g/dia, \%PV e g/dia/UTM foram, respectivamente, de 1.473,38; 3,94 e 97,43 (Tabela 3). Esse resultado indica que os ovinos apresentam mecanismos de adaptação a mudanças na deita, o que evitou a depreciação do consumo de matéria seca. As médias do consumo de proteína bruta (CPB) e do consumo de nutrientes digestíveis totais (CNDT) não diferiram entre os tratamentos e foram $233,51 \mathrm{~g} / \mathrm{dia}$ e $956,16 \mathrm{~g} / \mathrm{dia}$, respectivamente, provavelmente influenciada pela igualdade no consumo de matéria seca.

$\mathrm{O}$ valor médio observado para consumo de matéria seca no presente trabalho foi de $39,05 \mathrm{~g} / \mathrm{kg}$ de PV. Castro et al. (2002), avaliaram o efeito da frequência da suplementação de concentrado sobre o consumo de matéria seca em ovinos que recebiam apenas volumoso ou suplementados com 450,0g/dia de concentrado e encontraram aumento no consumo de matéria seca com o aumento da frequência de suplementação de uma para duas vezes ao dia. O consumo médio de matéria seca relatado por esses autores foi de $20,20 \mathrm{~g} / \mathrm{kg}$ de PV. Hadjigeorgiou et al. (2003), avaliaram a seleção, o consumo e a resposta digestiva de animais que recebiam apenas volumoso preparado em três comprimentos: longo, médio e curto, com os tamanhos médios de partículas, 13,$29 ; 7,26$ e $0,69 \mathrm{~mm}$, 
Rev. Bras. Saúde Prod. Anim., Salvador, v.13, n.1, p.137-149 jan/mar, 2012 http://www.rbspa.ufba.br ISSN 15199940

respectivamente, e observaram aumento no consumo de matéria seca com a redução do tamanho de partícula do volumoso em ovinos, com valores relatados em g/dia/UTM de 60,1; 61,1 e 66,2 nos tamanhos longo $(13,29 \mathrm{~mm})$, médio $(7,26 \mathrm{~mm})$ e curto $(0,69 \mathrm{~mm})$, respectivamente. No presente experimento o volumoso foi picado em quatro tamanhos (2mm; 5mm; $10 \mathrm{~mm}$ e $25 \mathrm{~mm}$ ) e fornecido separado do concentrado (colocados em compartimentos separados dentro do cocho, eram ofertados ao mesmo tempo). Assim, infere-se que esse manejo tenha estimulado o consumo de concentrado, o que por sua vez pode ter favorecido para igualdade nos tratamentos.

Tabela 3. Médias do consumo de matéria seca (CMS), de matéria orgânica (CMO), de proteína bruta (CPB), de extrato etéreo (CEE), de carboidratos não fibrosos (CCNF), de fibra em detergente neutro (CFDN), de fibra em detergente ácido (CFDA) e de nutrientes digestíveis totais (CNDT) nos diferentes tratamentos e coeficientes de variação $(\mathrm{CV})$

\begin{tabular}{|c|c|c|c|c|c|c|c|c|c|c|}
\hline \multirow{3}{*}{ Item } & \multicolumn{8}{|c|}{ Tratamentos } & \multirow{3}{*}{ Média } & \multirow{3}{*}{$\begin{array}{l}\text { CV } \\
(\%)\end{array}$} \\
\hline & \multicolumn{4}{|c|}{ Duas Refeições } & \multicolumn{4}{|c|}{ Quatro Refeições } & & \\
\hline & $2 \mathrm{~mm}$ & $5 \mathrm{~mm}$ & $10 \mathrm{~mm}$ & $25 \mathrm{~mm}$ & $2 \mathrm{~mm}$ & $5 \mathrm{~mm}$ & $10 \mathrm{~mm}$ & $25 \mathrm{~mm}$ & & \\
\hline $\mathrm{PV}(\mathrm{kg})$ & 38,35 & 36,77 & 35,63 & 38,74 & 41,01 & 36,43 & 38,44 & 36,45 & 37,72 & - \\
\hline UTM (kg) & 15,40 & 14,91 & 14,54 & 15,51 & 16,20 & 14,79 & 15,43 & 14,80 & 15,19 & \\
\hline CMS (g/dia)* & 1537 & 1470 & 1418 & 1515 & 1510 & 1552 & 1472 & 1309 & 1473,3 & 16,68 \\
\hline CMS $(\% \mathrm{PV})^{*}$ & 4,03 & 4,11 & 4,11 & 3,94 & 3,68 & 4,20 & 3,83 & 3,67 & 3,94 & 18,97 \\
\hline CMS (g/UTM)* & 100,1 & 100,4 & 99,3 & 98,0 & 93,2 & 103,3 & 95,3 & 89,5 & 97,43 & 17,31 \\
\hline CMO (g/dia)* & 1425 & 1362 & 1314 & 1403 & 1399 & 1437 & 1364 & 1212 & 1365,0 & 18,88 \\
\hline CPB (g/dia)* & 242,0 & 233,7 & 219,7 & 244,8 & 238,1 & 247,4 & 234,3 & 207,7 & 233,51 & 17,71 \\
\hline CEE (g/dia)* & 17,85 & 16,99 & 16,25 & 17,38 & 17,51 & 17,91 & 17,01 & 15,14 & 17,00 & 16,75 \\
\hline $\mathrm{CCNF}$ (g/dia)* & 411,2 & 396,6 & 373,3 & 414,6 & 404,4 & 419,6 & 397,6 & 352,5 & 396,26 & 17,62 \\
\hline CFDN (g/dia)* & 754,8 & 715,4 & 695,3 & 726,8 & 740,0 & 754,0 & 715,4 & 637,2 & 717,38 & 16,06 \\
\hline CFDN $(\% \mathrm{PV})^{*}$ & 1,97 & 2,00 & 2,01 & 1,89 & 1,80 & 2,04 & 1,85 & 1,78 & 1,92 & 18,19 \\
\hline CFDA (g/dia)* & 389,1 & 369,0 & 358,4 & 375,4 & 381,4 & 389,6 & 369,0 & 328,1 & 370,03 & 16,18 \\
\hline CNDT (g/dia)* & 1011 & 968 & 939 & 1048 & 963 & 967 & 924 & 825 & 956,16 & 18,19 \\
\hline
\end{tabular}

*Não houve diferença entre tratamentos (SNK; $p>0,05)$.

$\mathrm{PV}=$ peso vivo; $\mathrm{UTM}=$ unidade de tamanho metabólico $\left(\mathrm{kg} \mathrm{PV}^{0,75}\right)$.

Macedo Júnior et al. (2006) ao estudarem a influência de diferentes níveis de fibra em detergente neutro (FDN) dietético em ovelhas da raça Santa Inês, relataram consumo de matéria seca de $1.380,02 \mathrm{~g} / \mathrm{dia}$ e $74,25 \mathrm{~g} / \mathrm{dia} / \mathrm{UTM}$, e o consumo de matéria orgânica (CMO) de $70,95 \mathrm{~g} / \mathrm{dia} / \mathrm{UTM}$; consumo de proteína bruta (CPB) de $15,53 \mathrm{~g} / \mathrm{dia} / \mathrm{UTM}$; consumo de fibra em detergente neutro (CFDN) de 26,34g/dia/UTM e consumo de fibra em detergente ácido (CFDA) de
7,33g/dia/UTM, para a dieta que continha $20 \%$ de feno Coast-cross (Cynodon spp.). $\mathrm{O}$ consumo de fibra em detergente neutro observado foi de $1,92 \%$ do PV e $47,22 \mathrm{~g} / \mathrm{dia} / \mathrm{UTM}$, valores superiores ao citado por Forbes (1995) de $35 \mathrm{~g} / \mathrm{dia} / \mathrm{UTM}$ como o valor em que começaria a prevalecer os fatores reguladores do consumo de matéria seca em ovinos relacionados com 0 enchimento do trato gastrintestinal e o efeito físico da dieta. No entanto, o 
concentrado utilizado apresentou elevado teor de FDN (39,56\% da MS), valor este que correspondeu a $60,57 \%$ da FDN da dieta, esta fibra oriunda de subprodutos moídos (fonte de fibra não forrageira FFNF) que, consequentemente, apresentam taxa de passagem mais elevada e menor efeito físico sobre o consumo. O somatório desses efeitos também pode ter contribuído para igualdade no consumo de matéria seca.

Os diferentes tamanhos de partícula e a freqüência de alimentação não afetaram as variáveis de digestibilidade dos nutrientes $(\mathrm{P}>0,05)$, não havendo interação entre os tratamentos (Tabela 4).

Tabela 4. Médias da digestibilidade aparente (\%) da matéria seca (DAMS), da matéria orgânica (DAMO), da proteína bruta (DAPB), do extrato etéreo (DAEE), dos carboidratos não-fibrosos (DACNF), da fibra em detergente neutro (DAFDN), da fibra em detergente ácido (DAFDA) e o teor de nutrientes digestíveis totais (NDT, \%) nos diferentes tratamentos e coeficientes de variação $(\mathrm{CV})$

\begin{tabular}{|c|c|c|c|c|c|c|c|c|c|c|}
\hline \multirow{3}{*}{ Item } & \multicolumn{8}{|c|}{ Tratamentos } & \multirow{3}{*}{ Média } & \multirow{3}{*}{$\mathrm{CV}(\%)$} \\
\hline & \multicolumn{4}{|c|}{ Duas Refeições } & \multicolumn{4}{|c|}{ Quatro Refeições } & & \\
\hline & $2 \mathrm{~mm}$ & $5 \mathrm{~mm}$ & $10 \mathrm{~mm}$ & $25 \mathrm{~mm}$ & $2 \mathrm{~mm}$ & $5 \mathrm{~mm}$ & $10 \mathrm{~mm}$ & $25 \mathrm{~mm}$ & & \\
\hline DAMS* & 66,60 & 66,78 & 69,28 & 70,79 & 64,53 & 63,43 & 63,89 & 63,61 & 66,12 & 6,20 \\
\hline DAMO* & 70,13 & 70,26 & 72,05 & 73,78 & 68,13 & 66,47 & 66,97 & 67,29 & 69,38 & 5,50 \\
\hline DAPB* & 70,18 & 68,16 & 71,21 & 72,36 & 68,81 & 65,80 & 66,65 & 65,15 & 68,54 & 5,24 \\
\hline DAEE* & 47,82 & 44,77 & 53,30 & 43,42 & 43,29 & 43,72 & 40,32 & 48,48 & 45,64 & 21,21 \\
\hline DACNF* & 90,51 & 88,62 & 86,88 & 90,03 & 88,78 & 87,77 & 86,84 & 85,67 & 88,14 & 4,29 \\
\hline DAFDN* & 59,63 & 61,43 & 64,50 & 65,77 & 57,23 & 55,54 & 56,67 & 58,26 & 59,88 & 9,00 \\
\hline DAFDA* & 56,90 & 59,54 & 62,23 & 63,43 & 54,86 & 53,34 & 55,43 & 56,93 & 57,83 & 10,45 \\
\hline $\mathrm{NDT}^{*}$ & 65,74 & 65,72 & 67,19 & 68,84 & 63,77 & 62,21 & 62,55 & 63,00 & 64,88 & 5,11 \\
\hline
\end{tabular}

*Não houve diferença entre tratamentos (SNK; $>>0,05)$.

A digestibilidade aparente da matéria seca (DAMS) foi 63,43\% (quatro refeições e $5 \mathrm{~mm}$ ) e $70,79 \%$ (duas refeições e $25 \mathrm{~mm}$ ), a média observada foi de 66,12\%. Este valor de digestibilidade aparente da matéria seca pode ser considerado elevado $(>60,0 \%)$, o que sugere que o controle do consumo de matéria seca estaria baseado na densidade energética da dieta (VAN SOEST, 1994), fato esse que pode estar associado à elevada proporção de concentrado (75\%) nas dietas. Mesmo o concentrado apresentando subprodutos, a digestibilidade das dietas pode ser considerada alta. Macedo Junior et al. (2009) ao trabalharem com ovelhas gestantes e diferentes relações volumoso:concentrado não verificaram diferenças na digestibilidade aparente da matéria seca, matéria orgânica, fibra em detergente neutro e fibra em detergente ácido. As médias da digestibilidade aparente da matéria orgânica (DAMO), digestibilidade aparente da proteína bruta (DAPB), digestibilidade aparente do extrato etéreo (DAEE), digestibilidade aparente do consumo de carboidratos não fibrosos (DACNF), digestibilidade aparente da fibra em detergente neutro (DAFDN), digestibilidade aparente da 
fibra em detergente ácido (DAFDA) e o teor de nutrientes digestíveis totais (NDT) da dieta não diferiram entre os tratamentos, e apresentaram os valores de $69,38 \% ; 68,54 \% ; 45,64 \%$; $88,14 \% ; 59,88 \% ; 57,83 \%$ e $64,88 \%$, respectivamente. Pode-se inferir que essa igualdade possa estar relacionada à não alteração no consumo da matéria seca, uma vez que a digestibilidade é função direta do consumo da matéria seca (MACEDO JÚNIOR et al, 2010).

Ao avaliarem o efeito de frequência da suplementação com concentrado sobre a digestibilidade aparente dos nutrientes, Castro et al. (2002) observaram que o aumento da frequência de suplementação não afetou estas variáveis. Os valores médios encontrados para DAMS, DAMO e DAFDN foram, respectivamente, $56,8 \%, 58,8 \%$ e $47,1 \%$, valores inferiores aos do presente estudo. Hadjigeorgiou et al. (2003) observaram redução linear na digestibilidade aparente dos nutrientes com a redução do tamanho de partícula do volumoso em ovinos e relataram valores para a digestibilidade da matéria seca de $52,4 \% ; \quad 52,2 \%$ e $49,3 \%$, respectivamente, nos tamanhos longo $(13,29 \mathrm{~mm})$, médio $(7,26 \mathrm{~mm})$ e curto $(0,69 \mathrm{~mm})$. Para digestibilidade aparente da matéria orgânica foram encontrados os valores de $52,4 \% ; 537 \%$ e $51,0 \%$, respectivamente. Para a digestibilidade aparente da proteína bruta foram encontrados para os respectivos tamanhos de partícula, $48,2 \% ; 47,7 \%$ e $45 \%$, e para a digestibilidade aparente da fibra em detergente neutro os valores de 52,1\%; $53,3 \%$ e $47,5 \%$. O fato do concentrado do presente estudo apresentar subprodutos fibrosos também pode ter contribuído para igualdade entre os tratamentos, por favorecer $\mathrm{o}$ atendimento das necessidades de fibra no ambiente ruminal. Contudo, torna-se a ressaltar o alto valor médio na digestibilidade da matéria seca $(66,12 \%)$
Macedo Júnior et al. (2007) relataram, para dieta que continha $20 \%$ de feno Coast-cross (Cynodon spp.), valores superiores para a DAMS, DAMO e DAPB em $\%$ de 74,24; 75,87 e 74,95; e inferiores para a DAFDN e DAFDA em $\%$ de 55,78 e 50,75, respectivamente, em comparação com as dietas que continham teores mais elevados de feno. Ressalta-se que o concentrado utilizado por esses autores era composto por milho finamente moído e farelo de soja. Embora fosse esperado que a redução do tamanho de partícula do volumoso elevasse a taxa de passagem do alimento no trato gastrintestinal, o que acarretaria em aumento do consumo e da digestibilidade aparente da MS e dos nutrientes (VAN SOEST, 1994), efeitos estes não foram observados. Neste experimento, o menor tamanho de partícula do volumoso pode ter passado mais rapidamente pelo trato digestivo, sofrendo com menor intensidade os efeitos da digestão.

De acordo com Firkins (1997), as partículas com menor tamanho passam mais rápido pelo rúmen devido à sua maior densidade, bem como a menor resistência para atravessar o orifício retículo-omasal. Provavelmente, a baixa participação proporcional do volumoso na dieta do presente ensaio fez com que o processamento do mesmo, pela moagem, tivesse pouco efeito sobre os parâmetros totais de consumo de nutrientes.

Como citado anteriormente, o concentrado utilizado no presente estudo apresentou elevado teor de FDN que correspondeu a $60,57 \%$ da FDN da dieta, esta fibra oriunda de subprodutos moídos, que, consequentemente, apresentam taxa de passagem mais elevada e menor efeito físico sobre o consumo, e maior digestibilidade do que a FDN forrageira. Houve efeito do tamanho de partícula do volumoso sobre o tempo gasto em 
Rev. Bras. Saúde Prod. Anim., Salvador, v.13, n.1, p.137-149 jan/mar, 2012 http://www.rbspa.ufba.br ISSN 15199940

ócio (minutos/dia), ruminação (RUM) e com a mastigação total (MAST) $(\mathrm{P}<0,05)$. Sobre as demais variáveis estudadas não houve efeito nem do tamanho de partícula e nem da frequência de alimentação $(\mathrm{P}>0,05)$, conforme a Tabela 5 .

Tabela 5. Efeito de diferentes tamanhos de partícula do volumoso e da frequência de alimentação no comportamento ingestivo (minutos/dia) em ovinos

\begin{tabular}{lccccccr}
\hline $\begin{array}{l}\text { Variável } \\
\text { Comportamento } \\
\text { Ingestivo }\end{array}$ & \multicolumn{4}{c}{ Tamanho de partícula } & \multicolumn{2}{c}{ Frequência } & \\
\cline { 2 - 6 } & $2 \mathrm{~mm}$ & $5 \mathrm{~mm}$ & $10 \mathrm{~mm}$ & $25 \mathrm{~mm}$ & $\begin{array}{c}\text { Duas } \\
\text { Refeições }\end{array}$ & $\begin{array}{c}\text { Quatro } \\
\text { Refeições }\end{array}$ & Média \\
\hline ING. $\mathrm{H}_{2} \mathrm{O}$ & 8,33 & 8,33 & 12,83 & 9,16 & 10,16 & 9,16 & 9,66 \\
ING. MM & 27,08 & 17,91 & 22,66 & 21,25 & 19,66 & 24,79 & 22,22 \\
ÓCIO & $1044,5^{\mathrm{A}}$ & $868,3^{\mathrm{B}}$ & $924,3^{\mathrm{B}}$ & $901,6^{\mathrm{B}}$ & 941,9 & 927,5 & 934,70 \\
RUM & $214,58^{\mathrm{B}}$ & $358,75^{\mathrm{A}}$ & $282,83^{\mathrm{A}}$ & $326,66^{\mathrm{A}}$ & 278,91 & 312,50 & 295,70 \\
ING & 145,83 & 187,91 & 198,25 & 180,83 & 189,95 & 166,45 & 178,20 \\
MAST & $360,41^{\mathrm{B}}$ & $546,66^{\mathrm{A}}$ & $481,08^{\mathrm{A}}$ & $507,50^{\mathrm{A}}$ & 468,8 & 478,9 & 473,9 \\
\hline
\end{tabular}

Médias nos diferentes tamanhos de partícula seguidas de letras maiúsculas distintas na mesma linha diferem entre si pelo teste SNK $(\mathrm{P}<0,05)$. Médias nas diferentes dietas seguidas de letras minúsculas distintas na mesma linha diferem entre si pelo teste $\operatorname{SNK}(\mathrm{P}<0,05)$.

ING. $\mathrm{H}_{2} \mathrm{O}=$ tempo ingerindo água; ING. $\mathrm{MM}=$ tempo ingerindo mistura mineral; ÓCIO = tempo em ócio; $\mathrm{RUM}=$ tempo ruminando; $\mathrm{ING}=$ tempo ingerindo ração; MAST $=$ atividade mastigatória total (tempo ruminando + tempo ingerindo ração). Coeficiente de Variação (CV \%) ING $\mathrm{H}_{2} \mathrm{O}=105,37$; CV ING. MM = 60,71; CV ÓCIO = 10,97; CV RUM. $=25,51 ;$ CV ING. $=31,77 ;$ CV MAST $.=21,66$.

Foram obtidos com o tamanho de $2 \mathrm{~mm}$ os menores tempos gastos em minutos/dia com a ruminação e com a atividade mastigatória total, com as médias de 214,58 e 360,4 que corresponderam, respectivamente, a $68,79 \%$ e $72 \%$ dos tempos gastos com estas variáveis nos demais tratamentos. $\mathrm{O}$ efeito do tamanho de partícula sobre a atividade mastigatória total ocorreu devido à influência do tempo gasto com a ruminação sobre essa variável. As médias de tempos gastos em minutos/dia com a ruminação e com atividade mastigatória total foram 295,70 e 473,91, respectivamente. Notase, que somente esse tratamento apresentou diferença dos demais, o que evidencia a capacidade dos ovinos em manter o comportamento ingestivo com poucas alterações, mesmo quando reduzido a $5 \mathrm{~mm}$ o tamanho do volumoso. $\mathrm{O}$ fato das dietas apresentarem somente $25 \%$ de volumoso pode também ter contribuído para essa resposta. Os animais passaram em média $9,66 \mathrm{~min} /$ dia ingerindo água $\left(\mathrm{ING} \mathrm{H}_{2} \mathrm{O}\right) ; 22,22 \mathrm{~min} /$ dia ingerindo mistura mineral (ING $\mathrm{MM}$ ); $934,72 \mathrm{~min} / \mathrm{dia}$ em ócio e $178,20 \mathrm{~min} / \mathrm{dia}$ ingerindo alimentos (ING).

Hadjigeorgiou et al. (2003) observaram efeito $(\mathrm{P}<0,01)$ do tamanho de partícula do volumoso sobre as variáveis de tempos gastos com ingestão e ruminação. Foram encontrados os valores de $352,8 \mathrm{~min} /$ dia para o maior comprimento de partícula, $329,7 \mathrm{~min} /$ dia para o comprimento médio, $260,6 \mathrm{~min} /$ dia para o menor comprimento de partícula do volumoso e média de 314 , $36 \mathrm{~min} /$ dia para a variável tempo gasto com ingestão. $\mathrm{O}$ tempo gasto com ruminação foi de $547,2 \mathrm{~min} /$ dia no maior comprimento de partícula do volumoso, 
Rev. Bras. Saúde Prod. Anim., Salvador, v.13, n.1, p.137-149 jan/mar, 2012 http://www.rbspa.ufba.br ISSN 15199940

576,0 $\mathrm{min} /$ dia no comprimento médio, $627,84 \mathrm{~min} /$ dia no menor tamanho e média de 583,6min/dia. Ao contrário do esperado, esses autores observaram redução do tempo gasto com ruminação à medida que se aumentou o comprimento de partícula do volumoso. Não houve efeito $(\mathrm{P}>0,01)$ do tamanho de partícula sobre a atividade mastigatória total, que apresentou valor médio de 899,0 $\mathrm{min} /$ dia, superior ao encontrado no presente estudo. Apesar dos animais apresentarem diferença no tempo gasto com ruminação e mastigação total, não se observou influencia no consumo de matéria seca como discutido anteriormente. Esse resultado mais uma vez evidencia a capacidade de adaptação dos ovinos a mudanças no manejo alimentar.

$\mathrm{Na}$ Tabela 6 são apresentadas as relações entre as variáveis do comportamento ingestivo (ruminação, mastigação e ingestão) e o consumo de fibra em detergente neutro (CFDN), sendo encontrada interação entre o tamanho de partícula do volumoso e a frequência de alimentação $(\mathrm{P}<0,05)$.

Tabela 6. Relação entre o tempo ruminando (RUM, min/dia), ingerindo (ING, min/dia) e de atividade mastigatória total (MAST, min./dia) com o consumo de fibra em detergente neutro (CFDN, kg/dia) de diferentes tamanhos de partícula do volumoso em ovinos recebendo a dieta em duas freqüências de alimentação

\begin{tabular}{|c|c|c|c|c|c|}
\hline \multirow{2}{*}{ Relação } & \multicolumn{4}{|c|}{ Tamanho de partícula } & \multirow{2}{*}{ Média } \\
\hline & $2 \mathrm{~mm}$ & $5 \mathrm{~mm}$ & $10 \mathrm{~mm}$ & $25 \mathrm{~mm}$ & \\
\hline \multicolumn{6}{|c|}{ RUM./CFDN } \\
\hline Duas Refeições & $283,74^{\mathrm{Aa}}$ & $445,97^{\mathrm{Aa}}$ & $400,57^{\mathrm{Aa}}$ & $418,28^{\mathrm{Aa}}$ & 387,14 \\
\hline Quatro Refeições & $293,09^{\mathrm{Aa}}$ & $570,76^{\mathrm{Aa}}$ & $411,64^{\mathrm{Aa}}$ & $564,49^{\mathrm{Aa}}$ & 460,00 \\
\hline Média & $288,41^{\mathrm{B}}$ & $508,37^{\mathrm{A}}$ & $406,10^{\mathrm{A}}$ & $491,38^{\mathrm{a}}$ & \\
\hline \multicolumn{6}{|c|}{ ING./CFDN } \\
\hline Duas Refeições & $245,59^{\mathrm{Aa}}$ & $251,47^{\mathrm{Aa}}$ & $334,99^{\mathrm{Aa}}$ & $229,48^{\mathrm{Aa}}$ & 265,38 \\
\hline Quatro Refeições & $144,25^{\mathrm{Bb}}$ & $262,25^{\mathrm{Aa}}$ & $242,13^{\mathrm{Aa}}$ & $310,82^{\mathrm{Aa}}$ & 239,86 \\
\hline Média & $194,92^{\mathrm{B}}$ & $256,86^{\mathrm{AB}}$ & $288,55^{\mathrm{A}}$ & $270,15^{\mathrm{AB}}$ & - \\
\hline \multicolumn{6}{|c|}{ MAST./CFDN } \\
\hline Duas Refeições & $529,33^{\mathrm{Aa}}$ & $697,44^{\mathrm{Aa}}$ & $735,55^{\mathrm{Aa}}$ & $647,75^{\mathrm{Aa}}$ & 652,52 \\
\hline Quatro Refeições & $437,34^{\mathrm{Aa}}$ & $833,01^{\mathrm{Aa}}$ & $653,76^{\mathrm{Aa}}$ & $875,31^{\mathrm{Aa}}$ & 699,86 \\
\hline Média & 483,33 & 765,23 & 694,66 & 761,53 & - \\
\hline
\end{tabular}

Médias nos diferentes tamanhos de partícula seguidas de letras maiúsculas distintas na mesma linha diferem entre si pelo teste SNK $(\mathrm{P}<0,05)$. Médias nas diferentes dietas seguidas de letras minúsculas distintas na mesma coluna diferem entre si pelo teste SNK $(\mathrm{P}<0,05)$. Coeficiente de Variação $(\mathrm{CV} \%)$ ING./CFDN = 32,35; CV RUM./CFDN = 31,14; MAST./CFDN = 24,86.

Observa-se que na relação entre o tempo de ruminação (RUM - $\mathrm{min} / \mathrm{dia})$ e o CFDN (kg/dia) ocorreu efeito apenas do tamanho de partícula, pois se obteve no menor tamanho de partícula $(2 \mathrm{~mm})$ a menor relação média encontrada $(288,41 \mathrm{~min} / \mathrm{dia} / \mathrm{kg}$ de $\mathrm{CFDN})$, o que mostra que em consumo de fibra em detergente neutro semelhantes, os animais passaram menos tempo ruminando neste tratamento. Em média, os animais gastaram $423,48 \mathrm{~min} / \mathrm{dia} / \mathrm{kg}$ de consumo de fibra em detergente neutro. 
No tratamento com $2 \mathrm{~mm}$, os animais que receberam quatro refeições gastaram menos tempo ingerindo, já os animais ingerindo partículas com $10 \mathrm{~mm}$ e duas refeições apresentaram a maior relação ING/CFDN em relação aos demais animais. A média de tempo gasto ( $\mathrm{min} / \mathrm{dia}$ ) com ingestão por $\mathrm{kg}$ de CFDN foi de 252,62.

Os animais que foram alimentados com o tamanho de partícula com $2 \mathrm{~mm}$ apresentaram a menor relação entre a atividade mastigatória total (MAST $\mathrm{min} / \mathrm{dia})$ e o CFDN(kg/dia). Os animais gastaram, em média, $676,19 \mathrm{~min} / \mathrm{dia} / \mathrm{kg}$ de CFDN.

No presente trabalho, a redução do tamanho de partícula para $2 \mathrm{~mm}$ afetou o tempo gasto com a ruminação e, consequentemente, com a atividade mastigatória total, o que mostra a importância do tamanho de partícula do volumoso nestas variáveis fisiológicas. As características físicas da fibra, principalmente o tamanho de partícula, influenciam a ruminação e a atividade mastigatória, e o tamanho limite $1,18 \mathrm{~mm}$, correspondente às partículas que não atravessam o orifício retículoomasal em bovinos (MERTENS, 1997). Deve-se ressaltar que as características químicas do alimento também podem influenciar a atividade mastigatória, como por exemplo, o teor de lignina que aumenta a resistência da fração fibrosa à mastigação, o que aumenta o tempo necessário para a redução das partículas. A atividade mastigatória relaciona-se também com o tipo de animal, tamanho e idade do mesmo, ingestão de MS e com a técnica de medição adotada (MERTENS, 1997; FRANÇA et al., 2009). Isto pode explicar a diferença entre o tempo de atividade mastigatória total $/ \mathrm{dia} / \mathrm{kg}$ de CFDN $(676,19)$ observado e o valor sugerido como padrão (150min de atividade mastigatória total $/ \mathrm{kg}$ de $\mathrm{CFDN}$ ) para classificação da efetividade física das gramíneas.

Os tempos gastos com a ruminação e com a atividade mastigatória total em ovinos são diminuídos com a redução do tamanho de partícula do volumoso. O tamanho de partícula do volumoso e a frequência de fornecimento da dieta não afetam o consumo e a digestibilidade aparente dos nutrientes em ovinos. Assim, conclui-se que os ovinos apresentam mecanismos que evitam perdas no consumo e na digestibilidade aparente dos nutrientes, mesmo sob grandes mudanças nas características de alimentação (tamanho de partícula e frequência).

\section{REFERÊNCIAS}

ALVES, K.S.; CARVALHO, F.F.R.; VERAS, A.S.C.; FERREIRA, M.A.; COSTA, R.G.; SANTOS, E.P.; FREITAS, R.G.; SANTOS JÚNIOR, C.M.; ANDRADE, D.K.B. Níveis de energia em dietas para ovinos Santa Inês: Digestibilidade Aparente. Revista Brasileira de Zootecnia, v.32, n.6, p.1962-1968, 2003.

ARRUDA, D.S.R.; CALIXTO JÚNIOR, M.; JOBIM, C.C.; SANTOS, G.T. Efeito de diferentes volumosos sobre os constituintes sangüíneos de vacas da raça holandesa. Revista Brasileira de Saúde e Produção Animal [Online], v.9, n.1, p.35-44, 2008 .

ASAE. S424. Method of determining and expressing particle size of chopped forage materials by sieving. In Standards. American Society of Agricultural Engineers, St. Joseph, MI, 2001. 
Rev. Bras. Saúde Prod. Anim., Salvador, v.13, n.1, p.137-149 jan/mar, 2012 http://www.rbspa.ufba.br ISSN 15199940

CASTRO, T.; MANSO, T.; MANTECÓN, A.R. Effect of either once or twice daily concentrate supplementation of wheat straw on voluntary intake and digestion in sheep. Small Ruminant. Research, v.46, p.43-50, 2002.

DeVRIES, T.J.; VON

KEYSERLINGK, M.A.G.; BEAUCHEMIN, K.A. Frequency of feed delivery affects the behavior of lactating dairy cows. Journal Dairy Science, v.88, p.3533-3562, 2005.

DeVRIES, T.J.; VON

KEYSERLINGK, M.A.G. Time of feed delivery affects the feeding and lying patterns of dairy cows. Journal Dairy Science, v.88, p.625-631, 2005.

DOREAU, M.; MICHALETDOREAU, B.; GRIMAUD, P.; ATTI, N.; NOZIÈRE, P. Consequences of underfeeding on digestion and absorption in sheep Small Ruminant Research, v.49, p.289-301, 2003.

FRANÇA, S.R.L.; GONZAGA NETO, S.; PIMENTA FILHO, E.C.; MEDEIROS, A.N.; TORREÃO, J.N.C.; MARIZ, T.M.A.; COSTA, R.G.

Comportamento ingestivo de ovelhas Morada Nova no terço final de gestação com níveis de energia metabolizável na dieta. Revista Brasileira de Saúde e Produção Animal [Online], v.10, n.1, p.73-84, 2009.

FIRKINS, J.L. Effects of feeding nonforage fiber sources on site of fiber digestion. Journal Dairy Science, v.80, p.1426-1437, 1997.

FORBES, J.M. Voluntary food intake and diet selection by farm animals. Madison: CAB International, 1995. $532 \mathrm{p}$.
FURUSHO-GARCIA, I.F.; PEREZ, J.R.O.; OLIVEIRA, M.V.M.

Componentes corporais e órgãos internos de cordeiros Texel $\mathrm{x}$ Bergamácia, Texel x Santa Inês e Santa Inês puros, terminados em confinamento, com casca de café como parte da dieta. Revista Brasileira de Zootecnia, v.32, n.6, p.1992-1998, 2003.

FRANÇA, S.R.L.; GONZAGA NETO, S.; PIMENTA FILHO, E.C.; MEDEIROS, A.N.; TORREÃO, J.N.C.; MARIZ, T.M.A.; COSTA, R.G.

Comportamento ingestivo de ovelhas Morada Nova no terço final de gestação com níveis de energia metabolizável na dieta. Revista Brasileira de Saúde e Produção Animal [Online], v.10, n.1, p.73-84, 2009.

HADJIGEORGIOU, I.E.; GORDON, I.J; MILNE, J.A. Intake, digestion and selection of roughage with different staple lengths by sheep and goats. Small Ruminant Research, v.47, p.117-132, 2003.

KONONOFF, P.J.; HEINRICHS, A.J. The effect of corn silage particle size and cottonseed hulls on cows in early lactation. Journal Dairy Science, v.86, p.2438-2451, 2003.

LAMMERS, B.P.; BUCKMASTER, D.R.; HEINRICHS, A.J. A simple method for the analysis of particle sizes of forage and total mixed rations.

Journal Dairy Science, v.79, p.922928, 1996.

MACEDO JÚNIOR, G.L.; PÉREZ, J.R.O.; ALMEIDA, T.R.V. Influência de diferentes níveis de FDN dietético no consumo e digestibilidade aparente de ovelhas Santa Inês. Ciência e

Agrotecnologia, v.30, p.547-553, 2006. 
Rev. Bras. Saúde Prod. Anim., Salvador, v.13, n.1, p.137-149 jan/mar, 2012 http://www.rbspa.ufba.br ISSN 15199940

MACEDO JÚNIOR, G.L.; PEREZ, J.R.O.; ZANINE, A.M.; BORGES, I. Qualidade da fibra para a dieta de ruminantes. Ciência Animal, v.17, n.1, p.7-17, 2007.

MACEDO JÚNIOR, G.L.; PEREZ, J.R.O.; PAULA, O.J.; ALMEIDA, T.R.V.; ASSIS, R.M.; FRANÇA, P.M.; SILVA, V.B.; BORGES, I.; BAIÃO, A. A.F. Níveis de fibra em detergente neutro na alimentação de ovelhas Santa Inês gestantes. Arquivo Brasileiro de Medicina Veterinária e Zootecnia, v.61, n.1, p.196-202, 2009.

MACEDO JÚNIOR, G.L.; FERREIRA, M.I.C.; BORGES, I.; SILVA, V.B.; COUTO, J.R.L.; CAVALCATI, L.F.L. Consumo e digestibilidade aparente das frações fibrosas por ovelhas gestantes submetidas ou não à restrição nutricional. Revista Brasileira de Saúde Produção Animal [Online], v.11, n.1, p.179-192, 2010.

MERTENS, D.R. Creating a system for meeting the fiber requirements of dairy cows. Journal Dairy Science, v.80, p.1463-1481, 1997.

MICHALET-DOREAU, B.; DOREAU, $M$. Influence of drastic underfeeding on ruminal digestion in sheep. Animal

Research, v.50, n.6, p.451-462, 2001.

NATIONAL RESEARCH COUNCIL NRC. Nutrient requirements of sheep. 6.ed. Washington, D.C.: National Academy Press, 1985. 99p.

NATIONAL RESEARCH COUNCIL NRC. Nutrient requirements of small ruminants: sheep, goats, cervids, and new world camelids. Washington, D.C.: National Academic Press, 2007. $362 p$.
SILVA, D.J.; QUEIROZ, A.C. Análise de alimentos: métodos químicos e biológicos. Viçosa: Universidade Federal de Viçosa, 2002. 235p.

SILVA, H.G.O.; PIRES, J.A.V.; SILVA, F.F. Farelo de cacau (Theobroma cacao L.) e torta de dendê (Elaeis guineensis, Jacq) na alimentação de cabras em lactação: consumo e produção de leite. Revista Brasileira de Zootecnia, v.34, n.5, p.1786-1794, 2005

SILVA, H.G.O.; PIRES, J.A.V.; CUNHA, P.A. Digestibilidade de dietas contendo silagem de capim-elefante amonizado e farelo de cacau ou torta de dendê em ovinos Revista Brasileira de Zootecnia, v.36, n.2, p.499-506, 2007

VAN SOEST, P.J. Nutritional ecology of the ruminant. 2.ed. Ithaca: Cornell University Press, 1994. 476p.

VAN SOEST, P.J.; ROBERTSON, J.B.; LEWIS, B.A. Methods for dietary fiber, neutral detergent fiber, and nonstarch polysaccharides in relation to animal nutrition. Journal Dairy Science, v.74, p.3583-3597,1991.

WEISS, W.P. Energy prediction equations for ruminant feeds. In: CORNELL NUTRITION CONFERENCE FOR FEED MANUFACTURERS, 61., 1999, Ithaca. Proceedings... Ithaca: Cornell University, 1999. p.176-185.

ZANINE, A.M.; MACEDO JÚNIOR, G.L. Importância do consumo da fibra para nutrição de ruminantes. Revista Eletrônica de Veterinária REDVET ${ }^{\circledR}$, v. 7, n.2, 2006.

Data de recebimento: 06/04/2011

Data de aprovação: 02/12/2011 